\title{
Research in cardiac radiology: a European Society of Radiology white paper
}

\author{
ESR Executive Council 2009 • ESR Working Group \\ on Cardiac Imaging Research of the European Society \\ of Cardiac Radiology
}

Received: 6 April 2010 /Accepted: 6 April 2010 /Published online: 4 May 2010

(C) European Society of Radiology 2010

\begin{abstract}
The past, present and future status of the practice of cardiac imaging is discussed, especially as it relates to cardiac imaging research. Recommendations for the future development are given; these emphasize the importance of multidisciplinary collaboration.
\end{abstract}

Keywords Cardiac imaging - Research ·

Computed tomography $\cdot$ Magnetic resonance imaging . $\mathrm{PET} / \mathrm{CT}$

\section{Introduction}

The field of cardiac imaging has undergone a tremendous development during recent decades. Development of coronary angiography and subsequent introduction of noninvasive imaging techniques for imaging of the heart such as echocardiography, SPECT, PET, multidetector CT (MDCT) and MRI have resulted in more accurate diagnosis and monitoring of cardiovascular disease. The new technical developments have also changed the roles of radiologists and cardiologists both in the clinical imaging of the heart and in cardiac research. Radiologists have made major contribution into development of imaging methodology of MDCT and cardiac MR (CMR) and great strides are also occurring within PET/CT.

ESR Executive Council 2009 · ESR Working Group on Cardiac Imaging Research of the European Society of Cardiac Radiology $(\triangle)$ European Society of Radiology,

Neutorgasse $9 / 2$,

1010 Vienna, Austria

e-mail: communications@myESR.org
In the early days of cardiac imaging development, there has been a watershed area between radiology and cardiology [1]. While cardiac and coronary angiography were conceived and developed by radiologists, the role of the radiologist in the performances of cardiac catheterization and coronary angiography has progressively decreased. The role of the radiologist has been further diminished by the increasing importance of echocardiography as the main non invasive first line investigation of cardiac disease. This technique which although was initially shared between cardiology and radiology has become almost exclusively the domain of the cardiologist. The practice of cardiac imaging in 1960/ 1970s of 20th century was mostly centered on conventional radiography and angiography, but, in the last decade of noninvasive cardiac imaging techniques, MDCT and CMR have substantially challenged the pre-eminence of catheterization and angiography.

CMR imaging has been gradually assuming more importance in cardiovascular diagnosis; with this increase in importance, the role of the radiologist has been reactivated. In addition, introduction and constant development of cardiac CT has further increased the clinical role of radiologists. The new data that these techniques have produced have increased the interest in clinical cardiac image analysis which in turn has led to an enhanced interest in cardiac research among radiologists. Currently radiologists are still performing most of the cardiac CT and MRI examinations [2] but, more recently, cardiologists have become increasingly involved in these investigations. Consequently the cardiologist's role in cardiac $\mathrm{CT}$ and MR research is expanding. An analysis of the publication data in CMR has shown that radiologists initially authored most of the publications involving cardiac MR, but now the balance has shifted [2]. The 
current roles of radiologists in general and their involvement in cardiac imaging have been challenged by other specialities [3].

It was reported that the number of publications on cardiac MR imaging written by radiologists and written by cardiologists were essentially the same [4]. There were significantly more cardiac MR articles on developing techniques and assessment of surgical therapies published by radiologists, but cardiologists published significantly more articles on congenital heart disease, cardiomyopathy and heart failure. It was also found that radiologists used animals and combinations of volunteers and technical material more often in their studies than did cardiologists. On the other hand, more clinical trials and randomized controlled trials were published by cardiologists [4].

Similar results were found in the recent publication on research in cardiac CT. The number of journal articles on cardiac CT from 1996 to 2006 increased around 15 fold which is an exponential increase at a rate of $28 \%$ per year [5]. In general radiologists published more articles and research papers on cardiac CT than cardiologists, but this difference was marginal and varied from country to country. In the USA and Japan cardiologists were more active in cardiac research than radiologists, in Germany and the Netherlands the situation was reversed [6]. It is absolutely clear that active participation by radiologists in cardiac research is necessary to sustain the substantial role of radiology in the cardiac imaging arena.

Cardiologists have direct access to patients and consequently have better possibilities for preparation and organization of clinical trials. Radiologists however have preferential access to radiological equipment. They usually act as pioneers in development of new clinical applications in cardiac imaging. This fact should be used more actively, because research in clinical applications tremendously expands following their clinical adoption.

Historically, much of published radiology research has consisted of descriptions of a series of observations (i.e., descriptive research). With additional training, motivation, and corresponding infrastructure, any radiologist can take the next step toward participation in hypothesisdriven clinical research [6]. These options of hypothesisdriven research include: prospective studies, participation in a multicenter clinical trials, and assessment of advances in technology, contribution to or identification of the evidence base for clinical practice, costeffectiveness and outcomes analysis. Networks and databases are gaining prime importance in preparation and realization of multicenter clinical trials. Subspecialty Societies working together with the ESR have stimulated research and can be the focus for further collaborative research development.

\section{Barriers for successful research among radiologists}

According to the consensus conference organized by Academy of Radiology Research (The Academy), the American College of Radiology (ACR), the American Roentgen Ray Society (ARRS) and the Radiological Society of North America (RSNA) in 2003 the most commonly experienced barriers to success were: (a) lack of support from the dean; (b) time required to provide clinical service; (c) diminished income associated with doing research rather than clinical service; (d) lack of protected time for conducting research; (e) lack of appropriate space to support competitive research; (f) perception of the role of radiology in the institution as a service provider only; (g) misperception and poor communication within radiology, which causes lack of respect between clinical radiologists and investigators - both physicians and non-physicians; (h) cultural conflicts between departments; and (i) selection of residents who have little or no interest in research [4]. It also worth mentioning other factors which may play a part: there is often reluctance to co-operate with cardiologists; furthermore many radiologists do not have sufficient competence in the basics of cardiovascular disease.

\section{Strategies for the promotion of radiologists' contribution to cardiac research}

Institutional and departmental leadership that supports clinical and basic research is essential for productive cardiac research in the radiology department. The role of department leader is critical in setting a priority on advancing radiology research and maintaining a culture that respects research and researchers [4].

It is important that research efforts are rewarded with public recognition of success in obtaining funding and reaching study goals. This recognition will encourage research and research mentoring. In the case of unsuccessful applications researchers should be reassured that the experience they gained was still valuable. A structured mentoring program is necessary to provide this type of reassurance and guidance for future projects. In addition to support from department leaders and ongoing mentorship, researchers will only be successful if they are provided with allocated time to participate in research studies.

In terms of clinical practice, radiologists need to establish a section of cardiac imaging at a national level as well as developing a considerable practice in cardiological imaging at a department level. One individual in each large radiological group needs to be designated as the lead cardiac radiologist. That individual must be competent to fulfill that role and ideally would be actively engaged in 
research in the field [2]. This individual will co-ordinate training for residents and serve as a source of experience both in practice and in terms of research. Such an approach will provide radiologists involved in cardiac imaging with the necessary space for training and research and dedicated time for data analysis, image manipulation, reporting, etc; this will minimize inter- and interdisciplinary tensions.

It is crucial to increase radiologists' activities in basic cardiac imaging researching. These efforts need support not only from the professional radiological societies, but also from industry and requires good collaboration with fundamental scientists and medical physics.

\section{Education and attraction of young specialists}

It is recommended that comprehensive training in cardiac imaging should be an important part of every radiology residency program and therefore potentially attract residents to the field of cardiac radiology. To be successful in cardiac research radiologists should have extensive knowledge in diagnosis and treatment of cardiac diseases. Therefore it is important that radiology residents who would like to subspecialize in cardiac imaging be trained both in cardiac imaging and clinical cardiology. Dedicated time spent by a radiology resident in the cardiology clinic will not only provide insight into cardiovascular disease processes and the relevance of cardiac imaging results, but also forge collaboration between the two departments. Reciprocal opportunities within radiology should be made available to cardiologists in training.

All residents should be exposed to research training, including the knowledge of study design, methods, data management and statistics. Those residents who do not advance to an academic career will still gain valuable knowledge that will prepare them to better understand and more critically evaluate imaging based research. For those residents and radiologists who are willing to actively participate in research comprehensive research training and mentoring curriculum should be provided. The practice of performing a study with subsequent manuscript preparation can significantly enhance their general training and should be a basic component of research training.

Encouraging radiology residents to pursue a career in academic medicine is important. Research efforts by residents should be publically recognized and applauded by residency program directors. Rewarding residents by funding their participation in scientific meetings could be one of the many tools to attract residents to research during their training. It is hopeful that these experiences and successes will encourage residents to commit to a career which includes research.
Establishment of centres of excellence in cardiac imaging is needed for dissemination of knowledge in cardiac imaging research. Good example of advances in this direction is search and support of such centers for the ESOR Cardiac Fellowship program.

\section{Collaboration with clinical partners}

Collaboration with cardiologists, cardiac surgeons and other clinicians who routinely refer patients for cardiac imaging is essential for the success in both clinical practice and research [3]. Collaboration with cardiologists will allow the radiologist to better understand the fundamental questions to which their clinical colleagues need answer. Further, the cardiologist can provide insight into how analysis of a given imaging study will influence the management of a given patient. On the other hand, the radiologist can provide the cardiologist a detailed understanding of the acquisition, analysis and limitations of different imaging techniques. Working together, radiologists and cardiologists can determine the clinical questions worth investigating and the most appropriate protocol of image acquisition and analysis for hypothesis testing.

In terms of clinical practice and research, pediatric radiologists also need to define a section or to designate a specialist of cardiac imaging in children and young adults with congenital heart diseases in each pediatric centre. These individuals must collaborate with pediatric and adult cardiologists, who routinely refer children and (young) adults with congenital heart diseases for primary diagnosis and follow up imaging respectively with MDCT and CMR. It is especially important that pediatric radiologists with special knowledge on congenital heart disease collaborate with adult cardiac radiologists to offer optimum quality of MDCT and CMR in adults with sequelae of congenital heart diseases.

In addition collaboration with specialists and professional societies in nuclear medicine is necessary for the development of cardiac applications for, investigations such as SPECT-CT and PET-CT and for realization of research projects in molecular imaging.

\section{Professional societies}

One of the important roles of the professional societies is to promote education and further training as well as research and development in the field of cardiac radiology. There is a need for a trained pool of young investigators and a number of different funding mechanisms are required to support research training for residents and junior faculty members. Professional societies need to continue to encourage and support young researchers in cardiac radiology. Funding for research and for attendance at 
scientific meetings is an essential part of this support. Attendance at scientific meetings will not only provide an opportunity for young investigators to present their work, but also provide inspiration for new ideas of research. These new ideas are what will continue to motivate young investigators to strive for a productive research career.

Recently, the problem of promotion of research in cardiac radiology has attracted attention of most European professional radiological organizations, such as ESR, ECR, ESOR, ESCR, EIBIR, SCMR, ESPR and ESTI [7-13] and others.

The European Training Charter for Clinical Radiology and the curriculum for subspecialty training in cardiac radiology, prepared by ESCR under the supervision of the ESR, have been substantially updated and expanded, which, have in turn, stimulated the interest of young radiologists to cardiac imaging in general and to research in this field in particular.

There is a need to train and educate radiologists who have an expertise in the clinical and scientific aspects of cardiac imaging. This has been declared as a prime mission of European Society of Cardiac Radiology (ESCR). Annual congresses of ECR and ESCR offer a comprehensive educational program, covering all fields of cardiac imaging.

The ESR and ESCR offer exchange programs for fellowships on cardiac imaging to radiologists at an early stage of training. These fellowships organized through the European School of Radiology (ESOR), provide 3 months of intense modular training, reviewing at least 100 cardiac cases that require $\mathrm{CT}$ and/or MRI evaluation. The training is carried out in selected academic training centers in Europe. This training also offers the possibility of research training and mentorship in cardiac radiology.

The ESOR, educational partner of ESCR, organizes courses on Cardiac Cross-Sectional Imaging within the frame of its GALEN Advanced Courses, which are focused and articulated on recent advanced in radiological imaging, related to topics across cross-sectional imaging. The course is aimed at residents in their 4th or 5th year of training in radiology and recent board-certified radiologists. The program is structured in lecture series and interactive repetition seminars, assigned to internationally renowned European faculties. Every course is accompanied by a selfassessment test. Besides ESOR courses, different European professional organizations (for example, European Society for Magnetic Resonance in Medicine and Biology(ESMRMB) and academic centers offer a wide variety of teaching activities.

The European Institute for Imaging and Biomedical Research (EIBIR) regards cardiac imaging as one of the major directions for research, especially where it links in molecular imaging. The role of this organization is especially important for promotion of molecular imaging and PET/CT in cardiac imaging.

\section{Conclusion}

1. Cardiac imaging is an important and fast growing field of research in radiology.

2. Radiologists should keep and strengthen their basic and pivotal role in cardiac imaging research.

3. Activities of radiologists in cardiac research should be concentrated both on development and approbation of new technical applications and on clinical evidencebased radiology (i.e. multicentre clinical trials and outcome studies).

4. A particular focus should be directed to research in coronary and cardiac CT, imaging of atherosclerosis, molecular cardiovascular imaging and development of cardiac applications of PET/CT, SPECT/CT and MRI/ SPECT.

5. Better co-operation with other specialties including cardiologists, pediatric cardiologists, cardiac surgeons and nuclear medicine specialists is mandatory.

6. Joint efforts of leading European professional radiological societies - in particular, ESR/ECR, ESOR, ESCR, ESMRMB, EIBIR, SCMR, ESPR and ESTI-are necessary for boosting research in cardiac radiology. These efforts should be primarily targeted to young radiologists. Further development of fellowships in cardiac imaging will help to strengthen radiologist's position in cardiac imaging.

7. Establishment of European network of centers of excellence in cardiac radiology will help to promote interest of radiologists in cardiac imaging research and to achieve good quality and high impact of such research activities.

8. Radiological Societies should encourage and facilitate co-operation between centres that carry out cardiac imaging to develop a research network, which should be effective in generating grant funding for combined multicentre research projects. All these steps will lead in the future to the recognition of cardiac radiology as a distinctive subspecialty.

Acknowledgments This paper was established by the European Society of Radiology (ESR) and the ESR Working Group on Cardiac Imaging Research of the European Society of Cardiac Radiology (ESCR). The writing team was led by V. Sinitsyn (Moscow/RU), M. Rees (Gwynedd/UK), and A. Samarin (Tallinn/EE). The paper was approved by the ESR Executive Council in March 2010.

\section{References}

1. Higgins CH (2000) Cardiac imaging. Radiology 217:4-10

2. Gunderman RB, Weinreb JC, Borgstede JP et al (2007) The 2006 ACR forum: cardiovascular imaging: learning from the past, strategies for the future. J Am Coll Radiol 4:24-31 
3. ESR Executive Council 2009 \& European Society of Radiology (2010) The professional and organizational future of imaging. Insights Imaging 1:12-20

4. Miguel-Dasit A, Marti-Bonmati L, Sanfeliu P et al (2007) Cardiac MR imaging: balanced publication by radiologists and cardiologists. Radiology 242:410-416

5. Alderson PO, Bresolin LB, Becker GJ et al (2004) Enhancing research in academic radiology departments: recommendations of the 2003 consensus conference. Radiology 232:405-408

6. Itagaki MW, Suh RD, Goldin JG (2009) Cardiac CT research: exponential growth. Radiology 252:468-476
7. European School of Radiology http://www.myecr.org/esor

8. European Society of Radiology (Education) http://www.myecr. org/education

9. European Society of Cardiac Radiology http://www.escr.org

10. European Institute for Biomedical Imaging Research http://www. eibir.org

11. Society for Cardiovascular Magnetic Resonance http://www.scmr. org

12. European Society of Thoracic Imaging http://www.esti-society. org

13. European Society of Pediatric Radiology http://www.espr.org 